\title{
Mesons and Hybrids in a Relativistic Many Body Theory
}

\author{
Stephen R. Cotanch and Felipe J. Llanes-Estrada \\ Department of Physics, North Carolina State University, Raleigh NC 27695-8202
}

\begin{abstract}
Using a field theoretical, QCD inspired Hamiltonian formulated in the Coulomb gauge, relativistic many-body calculations are reported which reproduce the semiquantitative features of the observed meson and lattice glueball spectrums. Dynamical chiral symmetry breaking is achieved utilizing a BCS vacuum ansatz, yielding gap equations and realistic quark (and gluon) constituent masses and condensates. The excited hadron states are obtained by diagonalizing the effective Hamiltonian in a truncated Fock space using both the TDA and RPA. The meson spectrum, for a variety of spin and parities, is reasonably well described. In general, the two approaches differ minimally, except for the $\pi$ and $\eta$ where only the RPA yields correct properties and a Goldstone boson in the chiral limit. Our unified model also describes hybrids and a three-body TDA calculation predicts exotic and non-exotic hybrid states all above 2 $\mathrm{GeV}$. Our results are consistent with lattice and flux tube hybrid masses, suggesting that the recently observed $1^{-+}$exotics below $2 \mathrm{GeV}$ have an alternative, perhaps four quark, structure.
\end{abstract}

This work continues our comprehensive, many-body approach to hadron structure $[1,2]$, and reports the first, quasiparticle three-body calculation for hybrid mesons. The formulation is based upon approximating the exact QCD Hamiltonian in the Coulomb gauge by an instantaneous kernel having linear confinement with slope, $\sigma=0.18 \mathrm{GeV}^{2}$, specified by lattice. With the exception of the flavored current quark masses (here $m_{u}=m_{d}=5 \mathrm{MeV}, m_{s}=150 \mathrm{MeV}, m_{c}=1.2 \mathrm{GeV}$ ), our theory entails only one predetermined parameter, yet is capable of providing a unified description of the quark, gluon and combined sectors for both ground (vacuum) and excited (hadron) states. The model also rigorously preserves chiral symmetry yet exhibits dynamical chiral symmetry breaking through constituent masses from the gap equation. In this paper we summarize our previous hadron results and detail a new hybrid meson application to confront interesting exotic $J^{P C}=1^{-+}$resonances recently observed at BNL [3].

We briefly describe our model (consult refs. [1,2] for additional information). Our QCD effective Hamiltonian is

$$
H=\int d \mathbf{x} \Psi^{\dagger}(\mathbf{x})(-i \alpha \cdot \nabla+\beta m) \Psi(\mathbf{x})-\frac{1}{2} \int d \mathbf{x} d \mathbf{y} \rho^{a}(\mathbf{x}) V_{L}(|\mathbf{x}-\mathbf{y}|) \rho^{a}(\mathbf{y})
$$

CP549, Intersections of Particle and Nuclear Physics: $7^{\text {th }}$ Conference, edited by Z. Parsa and W. J. Marciano (C) 2000 American Institute of Physics 1-56396-978-5/00/\$17.00 


$$
-g_{s} \int d \mathbf{x} \Psi^{\dagger}(\mathbf{x}) \vec{\alpha} \cdot \mathbf{A}(\mathbf{x}) \Psi(\mathbf{x})+\operatorname{Tr} \int d \mathbf{x}(\mathbf{\Pi} \cdot \mathbf{\Pi}+\mathbf{B} \cdot \mathbf{B})
$$

involving both quark, $\Psi$, and gluon, $\mathbf{A}$, fields with color density $\rho^{a}=\Psi^{\dagger} \frac{\lambda^{a}}{2} \Psi+$ $f^{a b c} \mathbf{A}^{b} \cdot \Pi^{c}$. The linear interaction, $V_{L}=\sigma r$, is obtained from lattice studies and Regge phenomenology (for certain observables we supplement this with the canonical Coulomb potential $V_{C}=-\frac{\alpha_{s}}{r}$ with $\alpha_{s}=\frac{g_{s}^{2}}{4 \pi}$ ).

We first address the ground state and set $g_{s}=0$. The BCS ansatz produces uncoupled gap equations (Schwinger-Dyson equations) for both quark and gluon sectors. The generated dynamical mass is of order $100 \mathrm{MeV}$ for light quarks and roughly $800 \mathrm{MeV}$ for the gluons. These dressed quasiparticles, which in the vacuum form Cooper pairs $\left({ }^{3} P_{0}\right.$ condensates), are the constituent degrees of freedom for the excited states, or hadron spectrum. Next, we separately apply the $1 \mathrm{p}-1 \mathrm{~h}$ TDA truncation for the pure gluon and quark sectors. This generates glueballs [1] in broad agreement with lattice calculations and a meson spectrum [2] that adequately describes the known resonances, with a few exceptions, notably the pion. In particular, the TDA $\pi-\rho$ splitting is only $\approx 200 \mathrm{MeV}$ which is insufficient, and motivated our improved RPA formulation. The RPA yields a better vacuum, $|R P A\rangle$, containing correlations beyond the BCS Cooper pairs (in particular, pion pairs), such that $Q(R P A)|R P A\rangle=0$, where the RPA pion creator operator is $Q^{\dagger}=\sum_{i j}\left(X_{i j} q_{i}^{\dagger} \bar{q}_{j}^{\dagger}-Y_{i j} q_{i} \bar{q}_{j}\right)$. Here $q_{i}, \bar{q}_{i}$ are the rotated quasiparticle operators and $X_{i j}, Y_{i j}$ are the RPA wavefunction components obtained from the coupled equations of motion. For the pion state, $|\pi\rangle$, the equations are obtained via $\left\langle\pi\left|\left[H, Q^{\dagger}\right]\right| R P A\right\rangle=M_{\pi}\left\langle\pi\left|Q^{\dagger}\right| R P A\right\rangle$. Significantly in the chiral limit, $m_{q}=0$, and we compute $M_{\pi}=0$, corresponding to a massless Goldstone boson. For a non zero quark mass, $m_{q}=5 \mathrm{MeV}$, the $\pi-\rho$ mass difference is now a more realistic $600 \mathrm{MeV}$ indicating that chiral symmetry, in contrast to spin interactions, is the major source of mass splitting. Thus with only one parameter, $\sigma$, all the features of the meson spectrum, with the exception of the $\eta-\eta^{\prime}$ channel which requires flavor mixing, are semi-quantitatively reproduced. Our results are displayed in figure 1.

Similarly for the glueball spectrum as detailed more completely in ref. [1]. Using both meson and glueball input, we now focus on the combined quark and glue sectors and formulate hybrid mesons as a three-body system $(q \bar{q} g)$. We again use the BCS and TDA methods and diagonalize in an extended basis, $\left[[q \otimes \bar{q}]_{8} \otimes g\right]_{0}|R P A\rangle$, now involving quark color octet states. The wave equation is studied variationally with alternative, carefully constructed, ansätze in different angular momentum channels incorporating the constraints from transversality in the Coulomb gauge, consistent with Yang's theorem. We consider only the lowest intermediate angular momenta, $L_{-}(q \bar{q}$ orbital $), L_{+}((q \bar{q}) g$ orbital) and $S$ (total intrinsic quark spin) contributing to each $J^{P C}$ channel. Our RPA calculation did not differ significantly, as the chiral color octet current is not conserved (in contrast to the color singlet charge) by our Hamiltonian (or by full QCD). In spite of the challenging difficulties attending a relativistic, non-local three body problem, the diagonalization is feasible on a supercomputer using a Monte Carlo multidimensional integration al- 


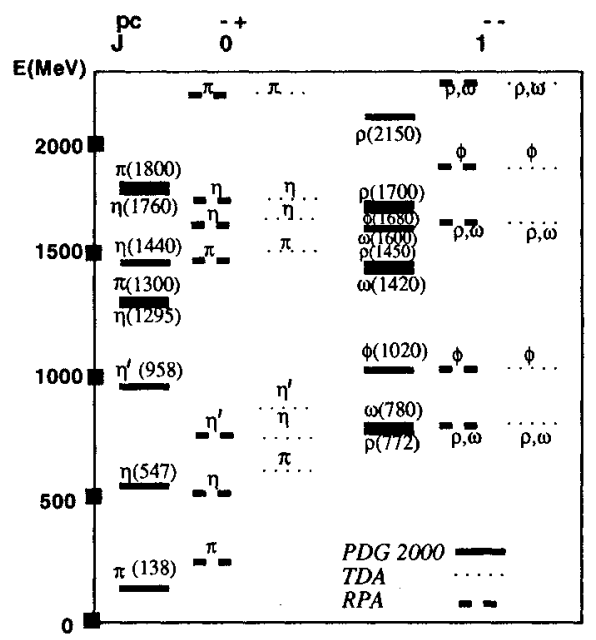

FIGURE 1. Calculations and data for the pseudoscalar and vector meson spectrum.

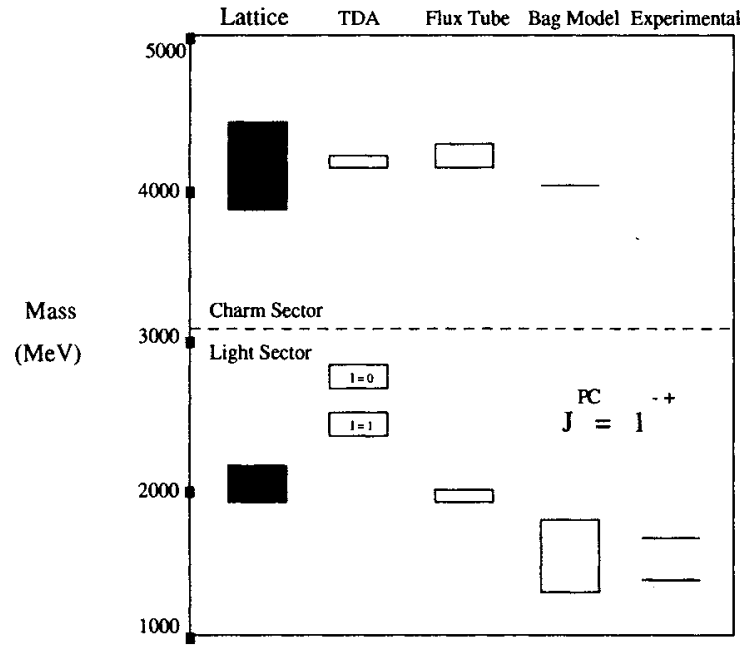

FIGURE 2. Comparison of different theoretical hybrid meson results in the exotic $1^{-+}$sector. 
gorithm and code Vegas. Further technical details will be reported in an upcoming extensive publication.

Our results are displayed in figure 2 and reveal a hybrid meson mass spectrum beginning in the low to mid $2 \mathrm{GeV}$ range. The reasons why our hybrids are much heavier than their valence $q \bar{q}$ counterparts are the large dynamical gluon mass and the repulsive interaction between the quark pair in a color octet. Another noticeable feature of figure 2 is the $I=1$ and 0 isospin splitting. This new effect, not present in color singlets, is due to quark and antiquark annihilation into a gluon for the spin 1, isospin 0 color octet state (analogous to annihilation in positronium). The process leads to a repulsive $q \bar{q}$ interaction, further raising the $I=0$ states. In quark channels coupled to spin 0 we recover the isospin degenerate spectrum.

Note in particular the exotic states $1^{-+}, 0^{--}, 3^{-+}$between 2 and $3 \mathrm{GeV}$. Each requires a $p$-wave, either $L_{+}$or $L_{-}$. Hence, the lightest exotic appears around 2.4 $\mathrm{GeV}$. This is somewhat higher, but still comparable to lattice. A comparison of the different theoretical models is also displayed in this figure along with the two controversial experimental states at 1.4 and $1.6 \mathrm{GeV}$ [3]. Consensus is growing, reflected by this figure, that these resonances are not hybrids but rather four quark states (meson resonances or quark molecules) which would have a lighter mass spectrum.

We also display in figure 2 our charmed hybrid exotic prediction and compare to other models which give similar results. Clearly, any new experimental data would be very enlighting as an excellent opportunity appears at hand for documenting gluonic degrees of freedom.

In conclusion, we have described mesons, glueballs and hybrids reasonably well with a unified model containing only one predetermined parameter. Our hybrid results, especially for the interesting exotic states, generally agree with other theoretical approaches and suggests an alternative structure for the observed $1^{-+}$states. Specifically, we submit these resonances may be four quark states. Our comprehensive approach readily extends to such systems and calculations are in currently in progress.

This work is supported by grants DOE DE-FG02-97ER41048 and NSF INT9807009. Supercomputer time is provided by NERSC.

\section{REFERENCES}

1. A. P. Szczepaniak, E. S. Swanson, C.-R. Ji, and S. R. Cotanch, Phys. Rev. Lett. 76, 2011 (1996).

2. F. J. Llanes-Estrada and S. R. Cotanch, Phys. Rev. Lett. 84, 1102 (2000) and references therein.

3. G. S. Adams et al. (E852 Collaboration) Phys. Rev. Lett. 81, 5760 (1998); D. R. Thompson et al. (E852 Collaboration) Phys. Rev. Lett. 79, 1630 (1997).

4. C. McNeile, hep-lat/9904013 (1999). 